\title{
Exploratory behaviors and recalibration: What processes are shared between functionally similar affordances?
}

\author{
John M. Franchak ${ }^{1}$
}

Published online: 25 May 2017

(C) The Psychonomic Society, Inc. 2017

\begin{abstract}
Recalibration of affordance perception allows observers to adapt to changes in the body's size or abilities that alter possibilities for action. Of key interest is understanding how exploratory behaviors lead to successful recalibration. The present study was designed to test a novel hypothesisthat the same processes of exploration and recalibration should generalize between affordances that share a similar function. Most affordances for fitting the body through openings are recalibrated without feedback from practicing the action; locomotion exploration is sufficient. The present study used a different fitting task, squeezing through doorways, to determine whether locomotor experience was sufficient for recalibrating to changes in body size that altered affordances. Participants were unable to recalibrate from locomotor experience, demonstrating that exploratory behaviors do not necessarily generalize between functionally similar affordances. Participants only recalibrated following action practice or after receiving feedback about judgment accuracy, suggesting that the informational requirements of the squeezing task may differ from those of other fitting tasks. Implications for affordance theory are discussed.
\end{abstract}

Keywords Affordances $\cdot$ Recalibration $\cdot$ Exploratory behavior $\cdot$ Practice $\cdot$ Feedback

An affordance refers to the fit between characteristics of the actor and the environment that make a particular action possible (Franchak \& Adolph, 2014a; Gibson, 1979; Stoffregen,

John M. Franchak

franchak@ucr.edu

1 Department of Psychology, University of California, Riverside, 900 University Avenue, Riverside, CA 92521, USA
2003; Warren, 1984). To choose actions adaptively and avoid motor errors, observers must perceive affordances - that is, detect perceptual information that distinguishes which actions are possible and impossible. The range of affordances available to humans is vast. Accordingly, researchers have investigated a wide array of affordances, including fitting through doorways (Fath \& Fajen, 2011; Higuchi et al., 2011; Petrucci, Horn, Rosengren, \& Hsiao-Wecksler, 2016; Thomas \& Riley, 2014; Warren \& Whang, 1987; Yasuda, Wagman, \& Higuchi, 2014), navigating under barriers (Franchak, Celano, \& Adolph, 2012; Stoffregen, Yang, Giveans, Flanagan, \& Bardy, 2009; Wagman \& Malek, 2009; Yu, Bardy, \& Stoffregen, 2011), sitting on seats (Mark, 1987; Mark, Baillet, Craver, Douglas, \& Fox, 1990; Stoffregen, Yang, \& Bardy, 2005), reaching (Thomas \& Riley, 2014; Thomas, Wagman, Hawkins, Havens, \& Riley, 2016), leaping and stepping (Cole, Chan, \& Adolph, 2013; Day, Wagman, \& Smith, 2015), throwing (Bingham, Schmidt, \& Rosenblum, 1989; Zhu \& Bingham, 2010), and catching (Oudejans, Michaels, Bakker, \& Dolne, 1996). Such studies reveal great diversity in the types of information that support affordance perception. For example, optic flow generated by postural movement allows observers to determine whether barriers of different heights are navigable (Stoffregen et al., 2009; Yu et al., 2011), haptic information from hefting balls of different sizes and densities supports perception of "throwability" (Bingham et al., 1989; Zhu \& Bingham, 2010), and optical time-to-contact information allows observers to detect if fly balls are catchable (Michaels \& Oudejans, 1992).

Affordance perception must be calibrated - that is, perceptual units must be scaled to action units such that perceptual information distinguishes between possible and impossible actions (Bingham \& Pagano, 1998; Withagen \& Michaels, 2005). For example, actors detect that doorways 1.1-1.3 times their shoulder width are possible to walk through, but smaller 
doorways require turning the body sideways to fit (Franchak et al., 2012; Warren \& Whang, 1987). Eye-height-scaled visual information relates doorway width to shoulder width, thus allowing actors to perceive fitting ability from a distance (Warren \& Whang, 1987). However, changes in affordances may disrupt calibration, such that perception no longer accurately detects affordances. Inaccurate perception means that observers either judge possible actions to be impossible or impossible actions to be possible. Holding a horizontal bar that is wider than the shoulders necessitates a revised calibration between eye height and body size (Wagman \& Taylor, 2005; Yasuda et al., 2014). Actors must recalibrate affordance perception by rescaling perceptual information to reflect new motor abilities. Recalibration occurs through the use of exploratory behaviors - actions that generate information about affordances. For example, postural movements generate eyeheight information through optic flow (Mark et al., 1990; Stoffregen et al., 2005; Stoffregen et al., 2009; Yu et al., 2011), and disrupting these postural movements prevents recalibration.

A widely studied aspect of calibration is how and when calibration transfers from one action to another. Findings reveal that calibration transfers between functionally similar actions but not between actions that are functionally dissimilar. For example, recalibration to altered speed of optic flow while walking transfers to crawling and to side-stepping but not to throwing (Rieser, Pick, Ashmead, \& Garing, 1995; Withagen \& Michaels, 2005). Similarly, altering the direction of optic flow while walking changes the calibration of walking but not the direction of throwing or kicking (Bruggeman \& Warren, 2010).

The present study tested a related but distinct question: Do the exploratory behaviors that recalibrate affordance perception generalize between functionally similar affordances? A novel hypothesis was tested: The function generalization hypothesis predicts that exploratory processes generalize between functionally similar affordances just as calibration transfers between functionally similar actions. ${ }^{1}$ For example, eye-height-scaled information from postural sway recalibrates affordances for fitting through both vertical openings (e.g., under barriers) and horizontal openings (e.g., doorways). This is consistent with the function generalization hypothesis, because both affordances are fitting actions and share the same exploratory process. Moreover, calibration of affordance perception for leaping transfers to perception of stepping, two functionally similar affordances (Day et al., 2015).

An alternative to the function generalization hypothesis is that functionally similar affordances require different types of exploratory behaviors to generate different types of perceptual

\footnotetext{
${ }^{1}$ Note that this hypothesis is independent of the question of whether calibration transfers between affordances; evidence supporting or disconfirming generalization of exploratory processes does not bear on the transfer of calibration.
}

information. In other words, variants of the same functional action depend on different variables for perceiving affordances. Consistent with this view, developmental studies show that affordance perception and exploration do not generalize between functionally similar affordances. What infants learn about crawling down steps does not generalize to walking down steps (Kretch \& Adolph, 2013), nor does learning about crawling down slopes transfer to walking down slopes (Adolph, 1995, 1997). Instead, learning is specific to the postural context, presumably because of differences both in the affordances (e.g., different body-environment relations for various forms of descent) as well in the exploratory behaviors that are used to perceive affordances (Adolph, 2008; Adolph, Joh, Franchak, Ishak, \& Gill-Alvarez, 2008).

The question of how exploratory processes generalize when perceiving different affordances addresses an important theoretical question: What level of description is appropriate for defining affordances? If the function generalization hypothesis is true, affordances can be meaningfully defined on the basis of function. For example, affordances for fitting the body through openings (e.g., walking through without touching the sides of the opening, squeezing through sideways, walking under an overhead barrier) would comprise a meaningful category if the exploratory processes that govern different variations of those actions are equivalent. Alternatively, if the fitting affordances are not equivalent, there may be a more detailed level at which such affordances must be defined.

The issue of taxonomy is not trivial. For observers to successfully recalibrate to changing affordances, they need to know how to explore to generate the requisite perceptual information. If exploratory processes generalize within functionally similar affordances, the demands on learning and development are less than in a scenario in which exploratory processes are more specialized. At this extreme - every variant of an action (e.g., turning to the right or to the left to navigate a doorway) necessitates different perceptual information and exploration - the task for observers would be intractable. Affordance researchers face an analogous problem in trying to understand how results generalize across affordance studies that use variants of functionally similar actions (e.g., stepping vs. leaping, overhead vs. lateral reaching).

\section{Recalibration and exploration in affordances for fitting}

The approach of the present study is to use a particular fitting action, squeezing through doorways, as a test case to determine whether the exploratory processes underlying recalibration are similar or different to functionally similar fitting actions that have been widely studied in past work.

Eye-height-scaled information, generated from postural movements and the resulting optic flow, allows observers to 
perceive affordances for fitting, such as walking through a doorway without turning (Warren \& Whang, 1987) and navigating under a barrier in a wheelchair (Stoffregen et al., 2009; $\mathrm{Yu}$ et al., 2011). Affordance judgments change when eye height is altered (Warren \& Whang, 1987). When adapting to new affordances, eye-height information from optic flow must be calibrated. Novice wheelchair users initially made inaccurate judgments of what barriers were possible to navigate under, but judgments improved following exploration using the wheelchair (Stoffregen et al., 2009). Critically, locomotor experience was sufficient for recalibration: Participants who navigated around in the wheelchair without practicing going under the barrier recalibrated equivalently to those who did practice wheeling under barriers. This finding suggests that action feedback - successful versus failed outcomes from practicing the action - is not necessary for recalibration in the barrier task. Mark and colleagues (1990) found a similar result in recalibration of affordances for sitting on seats after leg length was modified by wearing platform shoes. Practice sitting on seats while wearing the platform shoes did not improve recalibration beyond conditions that only included postural sway information, and practice did not permit accurate judgments when postural sway was eliminated. Note that throughout this article, practice will refer to practice the action as opposed to practicing making perceptual judgments.

The function generalization hypothesis predicts that another variant of the fitting task, squeezing through doorways, should be supported by the same exploratory processes. Specifically, locomotor experience should be sufficient for recalibration to changes in affordances for squeezing through doorways, and recalibration should not depend on action feedback from practice. Past work is inconclusive regarding the role of exploratory behaviors in the squeezing task, but it is clear that some type of exploration is needed for recalibration. In a recent study, participants judged whether they could squeeze through doorways while wearing a "pregnancy pack" that altered their squeezing ability (Franchak \& Adolph, 2014b). Initial judgments were inaccurate, averaging $10.5 \mathrm{~cm}$ of error. Twenty trials of practice squeezing through doorways facilitated recalibration by reducing errors to only $2.4 \mathrm{~cm}$. Practice effects on recalibration have also been observed in participants walking through doorways while holding a bar horizontally and judging whether it is possible to fit through without turning (Yasuda et al., 2014). Although these studies indicate that practice facilitates recalibration, conditions with locomotor experience without practice, as in Stoffregen and colleagues (2009), were not tested. One possibility is that practice only led to recalibration because it provided locomotor experience as participants walked back and forth through the apparatus, which would imply that action feedback was not required. This seems unlikely, as participants were unable to recalibrate when participants practiced walking through a single, large opening size but could recalibrate if practice involved doorways that provided information about both success and failure (Yasuda et al., 2014).

Alternatively, recalibration in the squeezing task may depend on practice if action feedback is required. Past work highlights a unique aspect of the squeezing task that differs from other fitting affordances: Squeezing through doorways depends not only on body size, but also on body compression (Comalli, Franchak, Char, \& Adolph, 2013; Franchak \& Adolph, 2014b; Franchak, van der Zalm, \& Adolph, 2010). Like friction (Joh, Adolph, Campbell, \& Eppler, 2006; Joh, Adolph, Narayanan, \& Dietz, 2007), compression is an emergent property: How much the body (or the body while modified by a backpack or pregnancy pack) compresses can only be understood given the opposing surface (like a doorway) and the amount of force applied. When squeezing through doorways, the body may compress by as much as $3-8 \mathrm{~cm}$ (Franchak, van der Zalm, Hartzler, \& Adolph, 2009). Practice squeezing through doorways may provide feedback about what doorway sizes permitted successful passage given body size and compression. Indeed, other types of affordances depend on action feedback for calibration. When learning to perceive throwability for balls varying in size and density, observers use information from hefting the balls (Bingham \& Pagano, 1998; Zhu \& Bingham, 2010). However, scaling hefting information to maximal throwing distance requires visual feedback about throwing distance. Participants who saw how far they threw during training were able to calibrate hefting behavior to judgments of throwability, but participants who did not have access to visual feedback of throwing were uncalibrated. Feedback may play a similar role in the doorway squeezing task calibrating visual information about doorway size relative to body size and compression.

Comparing different exploratory behaviors-locomotor experience versus practice - will test the function generalization hypothesis. However, a confound in past work is that visual information about the object used to alter participants' body dimensions in fitting tasks was always permitted. Participants had continual visual access to the pregnancy pack because it was worn on the front of the body (Franchak \& Adolph, 2014b). Similarly, participants could see the rod they held in their hands when making judgments about walking through doorways (Yasuda et al., 2014). Thus, it is unknown whether participants could have recalibrated without vision of the body-modifying object or whether visual access conferred any advantage. I predict that vision of the body-modifying object is not critical: Past work shows that affordance perception is independent of judgments of objects that alter affordances (Mark, 1987; Thomas \& Riley, 2014; Thomas et al., 2017). Moreover, participants were no worse at judging affordances for reaching with rods when rods were present in view or absent (Thomas \& Riley, 2014). Altering vision of the body-modifying object provides a way to determine what role, if any, it plays in perceiving affordances for squeezing through 
doorways, and controls for that information in testing the function generalization hypothesis.

\section{Present study}

The present study tested the hypothesis that exploratory behaviors generalize between functionally similar actions. A particular task-squeezing through doorways-was selected because it may differ from other fitting actions, such as navigating under barriers without ducking and walking through doorways without turning or squeezing. Participants wore a body-modifying object (a backpack) that altered their abilities to squeeze through doorways, and then they provided pretest judgments of their ability to squeeze through doorways of varying width. Participants then completed different types of exploratory behaviors (Table 1). Afterward, they made posttest judgments of squeezing ability. Each participant's actual ability threshold was determined so that a judgment error could be calculated. A reduction of judgment error served as evidence for successful recalibration. Failure to reduce error implied inaccurate affordance perception.

Participants were assigned to conditions that varied according to exploratory behaviors, which varied in the types of information that could be used to recalibrate perception (Table 1). In the practice front (PF) condition, participants wore the backpack on the front of the body and completed exploratory practice trials, replicating past work (Franchak \& Adolph, 2014b). To determine whether vision of the backpack is required for recalibration (and to justify eliminating vision of the backpack in the remaining conditions), the participants in the practice back (PB) condition completed the same

Table 1 Summary of the types of information available to the participants during exploration trials

\begin{tabular}{|c|c|}
\hline Condition & Available information \\
\hline PF (practice, front) & $\begin{array}{l}\text { Optic flow, haptic squeezing, vision of } \\
\text { backpack, visual feedback, haptic } \\
\text { feedback }\end{array}$ \\
\hline PB (practice, back) & $\begin{array}{l}\text { Optic flow, haptic squeezing, visual } \\
\text { feedback, haptic feedback }\end{array}$ \\
\hline WS (walking \& squeezing) & Optic flow, haptic squeezing \\
\hline AF (action feedback) & Visual feedback ${ }^{*}$ \\
\hline SE (squeezing, no vision) & Haptic squeezing, haptic feedback \\
\hline
\end{tabular}

Optic flow refers specifically to optic flow during locomotion. Optic flow while standing still was available throughout the study in every condition.

* Indicates mediated information about action feedback is supplied by the experimenter procedure while wearing the backpack on their backs. Participants were predicted to successfully recalibrate in both practice conditions.

Multiple types of information are available to participants while practicing (Table 1). In Stoffregen and colleagues' (2009) study, practice was no better than locomotor experience, suggesting that only some the information from practice, namely optic flow, was responsible for recalibration. Whether locomotor experience alone allows recalibration in the squeezing task is unknown, and is crucial for testing the function generalization hypothesis. Thus, participants in the walkingand-squeezing (WS) condition completed exploratory trials in which they walked while wearing the backpack and pressed it against a wall (not part of the doorway apparatus). Squeezing the backpack provided an opportunity to detect relevant haptic information about the backpack. Thus, the WS condition eliminated action feedback while preserving other relevant information, including optic flow information that had permitted recalibration in other fitting tasks (Stoffregen et al., 2009; Yu et al., 2011). The amount of walking and squeezing experience was $3.5 \mathrm{~min}$, which exceeded the amount of experience provided in Stoffregen and colleagues' (2009) investigation ( $2 \mathrm{~min}$ ). The function generalization hypothesis predicts that participants in the WS condition should recalibrate similarly to those in the PB condition.

The action feedback condition (AF) complemented the WS condition by providing only verbal feedback about the accuracy of participants' judgments while eliminating other information generated from practice (Table 1). Between pretest and posttest, participants in the AF condition made judgments about whether they could squeeze through different doorways and received feedback from the experimenter about whether those judgments were correct or incorrect. If action feedback is sufficient for recalibration, participants in the AF condition should recalibrate.

One methodological issue that arose in the AF condition is that abilities needed to be measured prior to exploration trials, so that the experimenter could provide true feedback. However, affordances are measured by having participants perform the action - that is, practicing - which would lead to recalibration (Franchak \& Adolph, 2014b; Yasuda et al., 2014). The solution was to have participants complete practice trials without any visual information at the start of the study. To verify that such experience did not lead to recalibration, a separate squeezing-experience-without-vision (SE) condition tested whether participants could recalibrate from squeezing through doorways with their eyes closed. This eliminated visual feedback while still providing haptic information from squeezing through the doorway, as well as haptic action feedback (feeling what doorways were possible to squeeze through). Because the focus of the investigation was on the accuracy of visual judgments, haptic exploration without 
visual information was predicted to be insufficient for recalibration. $^{2}$

\section{Method}

\section{Participants and design}

A power analysis was conducted using the results from Franchak and Adolph's (2014b) Experiment 3, because a similar design was employed in the present study. The reported effect size for the change from pretest to posttest was very large, partial $\eta^{2}\left(\eta_{\mathrm{p}}{ }^{2}\right)=.39$, meaning that a sample size of only six per group would be needed for $80 \%$ power. However, there was no guidance in the literature for testing the interactions between these different exploratory behaviors. Thus, to be conservative I adopted a medium effect size and determined that a sample size of 100 (20 per condition) would exceed $80 \%$ power.

Accordingly, 100 adults (48 males, 52 females) participated in the study. The participants were $18-26$ years old $(M=$ $20.0, S D=1.3$ ), were recruited from the psychology department subject pool, and received course credit for participation. One additional participant who was recruited did not complete the experiment due to a computer error. Participants were randomly assigned to one of five conditions (Table 1): practice with backpack worn on the front (PF), practice with backpack worn on the back (PB), walking and squeezing experience without feedback (WS), squeezing experience without vision (SE), or judgments with action feedback (AF).

\section{Apparatus}

An adjustable apparatus was constructed to present doorways of different widths (Fig. 1). The moving door $(185 \mathrm{~cm}$ tall $\times$ $100 \mathrm{~cm}$ wide) was attached to a trolley that moved within a free-standing metal frame $(213 \mathrm{~cm}$ tall $\times 280 \mathrm{~cm}$ wide $)$. A stationary wall $(182 \mathrm{~cm}$ tall $\times 62 \mathrm{~cm}$ wide) was fixed to the left side of the frame, perpendicular to the moving door. When open to its largest dimension, the doorway (space between the moving wall and the stationary wall) measured $70 \mathrm{~cm}$ in width. A camera attached to the moving door displayed calibration markings to allow the experimenter to set the doorway width in $0.5-\mathrm{cm}$ increments. A locking mechanism fixed the doorway at a particular width so that it stayed the same width while participants attempted to squeeze through. Participants began each trial at a starting line marked on the floor $320 \mathrm{~cm}$ away from the doorway. A camera mounted to the top of the doorway frame allowed the experimenter to monitor the participants.

\footnotetext{
${ }^{2}$ It is possible that the SE condition might recalibrate haptic judgments; however, testing haptic judgments was beyond the scope of this investigation.
}

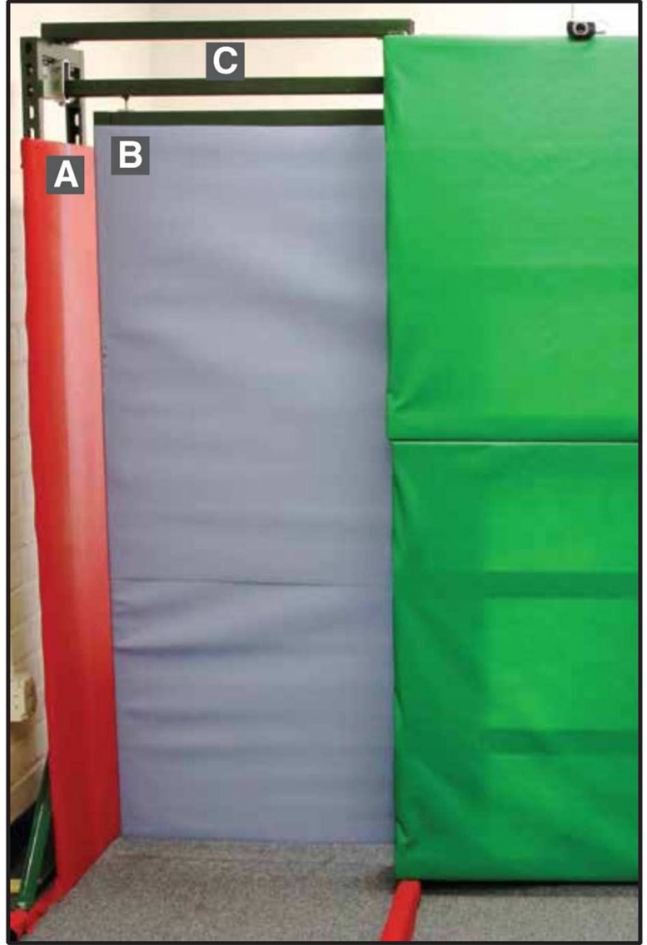

Fig. 1 Apparatus with adjustable doorway. A stationary wall (A) and a sliding wall (B) formed the doorway opening. The sliding wall hung on a frame within a sliding track $(\mathrm{C})$.

Participants wore a backpack $(43 \mathrm{~cm}$ tall $\times 25 \mathrm{~cm}$ wide $\times$ $12 \mathrm{~cm}$ deep) that weighed $1.1 \mathrm{~kg}$. The backpack was filled with corrugated cardboard to ensure that it stayed rigid as they squeezed through the doorway. Waist and sternum straps were used to keep the backpack properly positioned on participants' bodies.

\section{Procedure}

In all conditions, participants began the study by putting on the backpack. In the practice front condition (PF), participants wore the backpack such that the bulk of the pack rested on the abdomen. In all other conditions (PB, WS, SE, and AF), participants wore the pack normally on their backs (the backpack fit against the middle of the back, above the buttocks). The participants in all conditions were given an opportunity to hold and inspect the backpack before putting it on. Although participants wearing the backpack on their backs may have been able to catch a glimpse of the backpack while rotating their heads all the way to one side, this would not allow them to view the backpack and the doorway simultaneously. Crucially, the participants in the PF condition had continual visual access to the backpack while making judgments, whereas the participants in the other conditions did not. Participants completed four sets of trials in the following order: 30 pretest judgment trials, 20 exploration trials, 30 posttest judgments trials, and 15 baseline ability measurement 
trials. Participants were aware that judgment accuracy would be tested before and after exploration.

Judgment trials For the pretest and posttest judgment trials, participants were instructed to say "yes" if they thought that they could successfully squeeze through the doorway in a sideways position - specifically, entering the doorway with the left shoulder - and to say "no" if they did not think they could get to the other side in this manner. To keep participants from seeing the doorway adjustments, participants started each trial facing away from the doorway and were cued to turn and make a decision after the experimenter had set the doorway to a particular width. Participants were allowed to move in place however they wished while making the judgments, as long as they stayed behind the starting line; this allowed postural sway that could generate visual information throughout the task. No feedback was given regarding judgment accuracy during judgment trials.

A custom MATLAB script determined the doorway width for each trial in order to find each participant's judgment threshold - the doorway size the participant deemed possible to squeeze through on $50 \%$ of trials - for each set of judgment trials. Three successive subsets of trials narrowed in to find the judgment threshold, which could vary widely between participants of different affordances and levels of perceptual accuracy. The first six trials used a binary search procedure (Franchak et al., 2010) between 15 and $70 \mathrm{~cm}$ (in $0.5-\mathrm{cm}$ increments) to find an initial threshold estimate. The doorway widths for the next 14 trials were presented at prespecified widths relative to the initial estimate: two trials each at $-5,-$ $4,-3,0,+3,+4$, and $+5 \mathrm{~cm}$ relative to the initial estimate (rounded to the nearest $0.5-\mathrm{cm}$ increment). The 14 trials were presented in random order. Afterward, a second threshold estimate was calculated (on the basis of all 20 judgments trials) and was used to determine the final ten trials: two trials each at $-2,-1,0,+1$, and $+2 \mathrm{~cm}$ relative to the second threshold estimate, again presented in a random order.

Exploration trials Exploration trials varied according to the test condition. For the PB and PF conditions, the exploration trials consisted of 20 practice trials. For each practice trial, the experimenter set the doorway to a particular size and then instructed the participant to attempt to squeeze through sideways. As in the judgment trials, the doorway was adjusted while the participant faced away. Participants received a range of doorway sizes to ensure that they sometimes successfully squeezed through and at other times failed to squeeze through. Participants were instructed to attempt to squeeze through the doorway even if they believed it was impossible. The experimenter did not provide explicit feedback. However, action feedback was apparent to the participant (i.e., they either made it through the doorway or became stuck, and thus were allowed to see which doorways permitted success and failure).
In addition to providing the participant with practice experience, walking practice trials were used to measure participants' backpack ability threshold - the doorway size that participants could successfully squeeze through on $50 \%$ of trials (Franchak \& Adolph, 2014a). Doorway sizes were determined by a MATLAB script that used a four-down/three-up staircase procedure (Cornsweet, 1962) starting from $32 \mathrm{~cm}$ to find the backpack ability threshold (see Calculation of Threshold Estimates for additional details).

Participants in the WS condition completed 20 exploration trials, during which they walked from the starting line to a wall in the experiment room, squeezed the backpack against the wall, and then returned to the starting line. The distance between the starting line and the wall was $320 \mathrm{~cm}$, identical to the distance between the starting line and the doorway apparatus. WS trials were conducted in the same room as the doorway apparatus; however, participants walked perpendicular to the apparatus, and the doorway remained closed. After completing the exploration and posttest trials, participants completed an additional 20 trials of walking through doorways (identical to the practice exploration trials in the $\mathrm{PB}$ and $\mathrm{PF}$ conditions), to provide data for calculating backpack ability thresholds. Presenting these practice trials following all judgments ensured that judgment accuracy was not affected by practice.

Participants in the SE condition completed 20 exploration trials in which they squeezed through the doorway without vision, and thus without visual feedback regarding which doorway widths would permit success and failure. Only haptic feedback was available. Participants stood one step away from the doorway. The experimenter cued the participant to close his or her eyes, then adjusted the doorway to the appropriate size and locked the door. Then the experimenter cued the participant to step through the doorway. Participants used their hands to feel to the other side of the doorway to guide themselves through. Once the participant had reached the other side of the doorway or become stuck, the experimenter opened the doorway and then told participants to open their eyes and return to the starting position. Data from successful and failed attempts to squeeze through the doorway were recorded and used to calculate backpack ability thresholds. Doorway widths for the 20 exploration trials were determined in the same way as for the exploration trials in the practice conditions.

Participants in the AF condition made judgments during exploration trials and received verbal feedback from the experimenter about judgment accuracy. Participants stood at the starting line and made judgments while viewing doorways of different widths. After each judgment, the experimenter said "correct" if the participant responded "no" to a doorway width smaller than the backpack ability threshold, or "yes" to a doorway width larger than the backpack ability threshold. In the reverse cases (participant said "yes" to a doorway smaller than the ability threshold or said "no" to a doorway 
larger than the ability threshold), the experimenter said "incorrect." Note that the participants in the AF condition did not receive feedback during the pretest and posttest judgment trials; feedback was only provided to judgments made during the exploration trial set.

For AF trials, the experimenter needed to know the participant's backpack ability threshold before the exploration trials in order to provide accurate judgment feedback. But if participants completed practice trials at the start of the session, experience from this practice would result in recalibration and preclude testing the effects of feedback. However, pilot testing showed that the SE condition - squeezing through doorways with eyes closed - did not result in recalibration, providing a way to measure participants' abilities without allowing them to learn. Thus, the participants in the AF condition completed 20 trials of squeezing through doorways with eyes closed before they completed the pretest judgments, so that ability thresholds could be calculated.

\section{Baseline measurement trials and body measurements} After completing all judgment and exploration trials, participants took off the backpack, and their baseline ability threshold was measured by walking through the doorway 15 times. The procedure was identical to that in the exploratory practice trials, except that participants squeezed through the doorway without wearing the backpack. Finally, each participant's body height and weight were measured (without the backpack). Due to a clerical error, height and weight measurements were not obtained from one participant in the front condition. In total, the experiment lasted $\sim 45 \mathrm{~min}$.

\section{Calculation of threshold estimates}

After the experiment, psychophysical functions were fit to the data in each set of trials to determine threshold estimates: Pretest judgment thresholds were calculated from the proportions of "yes" responses in pretest judgment trials, backpack ability thresholds were calculated from the proportions of successes in walking practice (or SE exploration) trials, posttest judgment thresholds were calculated from the proportions of "yes" responses in the posttest judgment trials, and baseline ability thresholds were calculated from the proportions of successes when walking through doorways without the backpack. The Palamedes Toolbox (Kingdom \& Prins, 2010) was used to fit cumulative Gaussian probability distribution functions to each participant's data from each set of trials using maximum likelihood estimation of the alpha (threshold) and beta (standard deviation) parameters (Wichmann \& Hill, 2001a, 2001b). Parametric bootstraps were performed with 500 Monte Carlo iterations to determine $95 \%$ confidence intervals for the threshold estimates. Figure 2 shows the curve fits and threshold estimates obtained from one participant's judgment

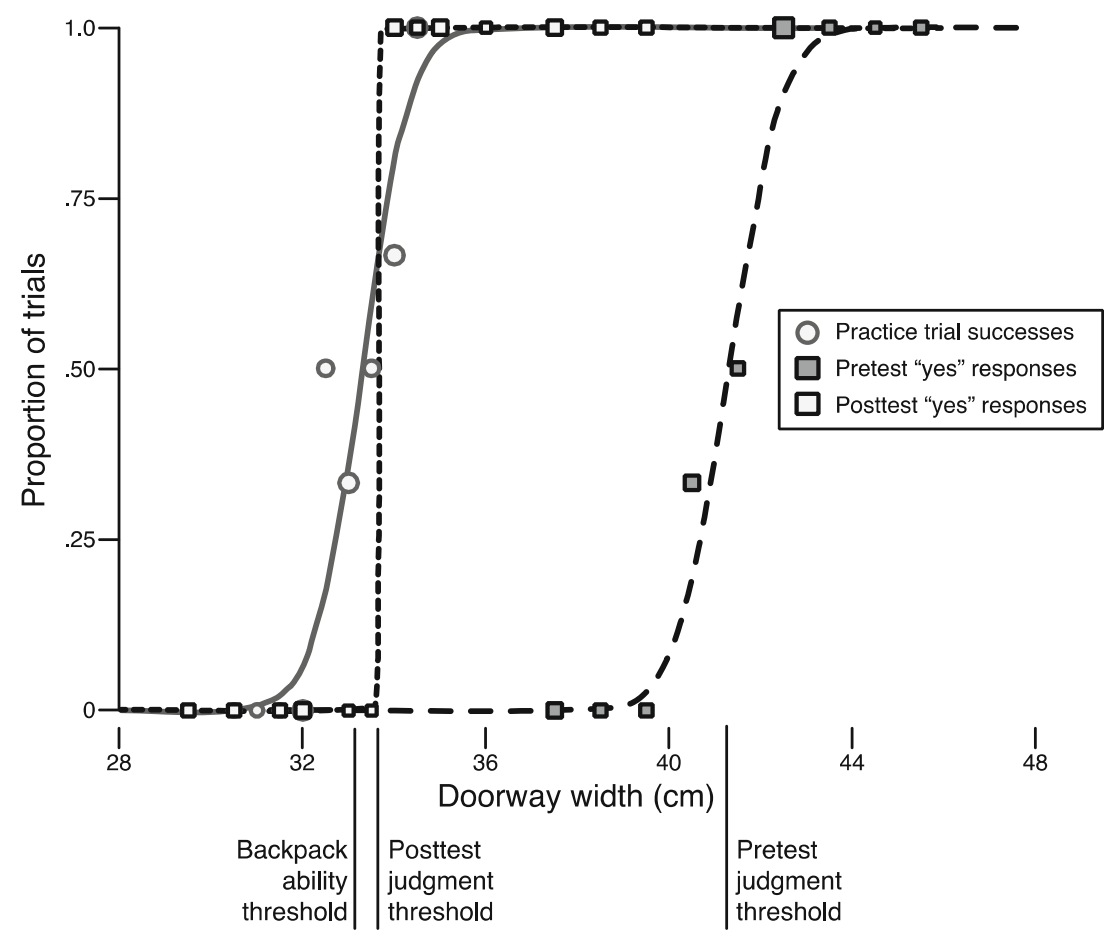

Fig. 2 Example ability and judgment data from one participant in the front condition. Circles show the participant's ability to fit successfully through doorways of different widths during the practice trials. A psychophysical function was fit to the success rates (gray line) to determine the backpack ability threshold (doorway width at the $50 \%$

point of the function). Functions were fit to the pretest judgments (gray squares) and the posttest judgments (white squares) to calculate the pretest judgment thresholds and posttest judgment thresholds using the same procedure. 
and ability data while wearing the backpack in the PF condition. The small confidence intervals found across each type of threshold in every condition (Table 2) indicate robust function fits and threshold estimates. Standard deviation parameter estimates were not sufficiently robust, given the small number of trials collected in each condition, so variable error was not analyzed.

\section{Calculation of dependent measures}

Pretest judgment error was calculated as the absolute value of the difference between each participant's pretest judgment threshold and backpack ability threshold. Similarly, posttest judgment error was defined as the absolute value of the difference between each participant's posttest judgment threshold and backpack ability threshold. To determine how much the backpack manipulation altered participants' abilities, manipulation size was calculated by subtracting each participant's baseline ability threshold from the backpack ability threshold.

\section{Results}

\section{Body measurements and manipulation size}

No significant differences were found in height, weight, or body-mass index between conditions (one-way ANOVA $p$ s $>$.17). Unexpectedly, manipulation size - the difference between ability thresholds with and without the backpack- differed across conditions (Table 3). Specifically, wearing the backpack on the front of the body in the PF condition altered affordances by a larger degree than did wearing the backpack on the back in the other conditions, $F(4,98)=12.11, p<.001$, $\eta_{\mathrm{p}}{ }^{2}=.340$. Sidak-corrected pairwise comparisons confirmed that the manipulation size in the PF condition was greater than

Table 2 Means and 95\% confidence intervals of the threshold estimates for each condition (all values in centimeters)

\begin{tabular}{lllll}
\hline Condition & $\begin{array}{l}\text { Pretest } \\
\text { Judgments }\end{array}$ & $\begin{array}{l}\text { Posttest } \\
\text { Judgments }\end{array}$ & $\begin{array}{l}\text { Backpack } \\
\text { Ability }\end{array}$ & $\begin{array}{l}\text { Baseline } \\
\text { Ability }\end{array}$ \\
\hline PF (practice, front) & $32.8 \pm 0.93$ & $31.3 \pm 0.78$ & $31.9 \pm 0.47$ & $21.1 \pm 0.22$ \\
PB (practice, back) & $32.4 \pm 1.21$ & $27.4 \pm 0.80$ & $28.5 \pm 0.49$ & $20.6 \pm 0.13$ \\
$\begin{array}{c}\text { WS (walking \& } \\
\text { squeezing) }\end{array}$ & $32.5 \pm 1.02$ & $33.2 \pm 0.81$ & $28.4 \pm 0.73$ & $20.9 \pm 0.12$ \\
$\begin{array}{c}\text { SE (squeezing, no } \\
\text { vision) }\end{array}$ & $30.6 \pm 0.85$ & $24.6 \pm 0.79$ & $30.0 \pm 0.54$ & $21.6 \pm 0.11$ \\
$\begin{array}{c}\text { AF (action } \\
\text { feedback) }\end{array}$ & $20.4 \pm 0.67$ & $26.2 \pm 0.74$ & $25.2 \pm 0.77$ & $18.2 \pm 0.06$ \\
\hline
\end{tabular}

Table 3 Means and standard deviations for manipulation size, pretest error, and posttest error for each condition

\begin{tabular}{llll}
\hline Condition & $\begin{array}{l}\text { Manipulation } \\
\text { Size }(\mathrm{cm})\end{array}$ & $\begin{array}{l}\text { Pretest Error } \\
(\mathrm{cm})\end{array}$ & $\begin{array}{l}\text { Posttest Error } \\
(\mathrm{cm})\end{array}$ \\
\hline PF (practice, front) & $10.8(1.3)$ & $5.54(5.08)$ & $2.01(1.49)$ \\
PB (practice, back) & $7.9(2.2)$ & $7.03(5.92)$ & $2.72(2.03)$ \\
WS (walking \& squeezing) & $7.9(2.0)$ & $7.99(6.17)$ & $6.80(6.85)$ \\
SE (squeezing, no vision) & $8.3(1.5)$ & $6.00(3.79)$ & $6.58(4.74)$ \\
AF (action feedback) & $7.0(2.1)$ & $6.65(2.66)$ & $2.14(2.34)$ \\
\hline
\end{tabular}

in all other conditions $(p s<.001)$ and that the remaining conditions did not differ significantly from one another ( $p s>$ $.35)$.

\section{Judgment error}

Irrespective of condition, participants were divided according to the types of errors they made at both pretest and posttest (Fig. 3). Some participants made risky decisions by saying "yes" to impossible doorways (thresholds to the left of the vertical line), and others were cautious and said "no" to possible doorways (thresholds to the right of the vertical line). This variation in error directions demonstrates why it was necessary to calculate absolute error: Averaging constant (signed) errors would result in risky and cautious errors cancelling out, making it impossible to detect whether accuracy changed from pretest to posttest in the different conditions.

Judgments were inaccurate during the pretest across all five conditions (Fig. 4, Table 3). Reductions in judgment errors from pretest to posttest - recalibration-occurred only in the conditions in which participants generated action feedback or received verbal feedback (PF, $\mathrm{PB}$, and $\mathrm{AF}$ ). In contrast, participants who received locomotor and haptic (WS) or only haptic (SE) experience without visual feedback did not recalibrate. A Condition $(\mathrm{PB}, \mathrm{PF}, \mathrm{WS}, \mathrm{SE}, \mathrm{AF}) \times$ Phase (pretest, posttest) ANOVA on judgment errors revealed main effects of phase, $F(1,95)=29.51, p<.001, \eta_{\mathrm{p}}{ }^{2}=.237$, and condition, $F(4,95)=3.01, p=.022, \eta_{\mathrm{p}}{ }^{2}=.112$. A significant Phase $\times$ Condition interaction indicated that condition moderated whether participants improved from pretest to posttest, $F(4$, $95)=4.27, p=.003, \eta_{\mathrm{p}}{ }^{2}=.152$. Sidak-corrected pairwise comparisons confirmed that errors decreased from pretest to posttest for the $\mathrm{PF}, \mathrm{PB}$, and $\mathrm{AF}$ conditions $(p \mathrm{~s}<.001)$, but not for the WS and SE conditions ( $p s>.26$ ). Additionally, Sidakcorrected pairwise comparisons did not reveal differences between pretest accuracy among the conditions ( $p s>.71)$. However, posttest errors were significantly smaller in the PF, $\mathrm{PB}$, and $\mathrm{AF}$ conditions than in the WS and SE conditions ( $p \mathrm{~s}<$ .03). Posttest errors did not differ between the PF, PB, and AF 

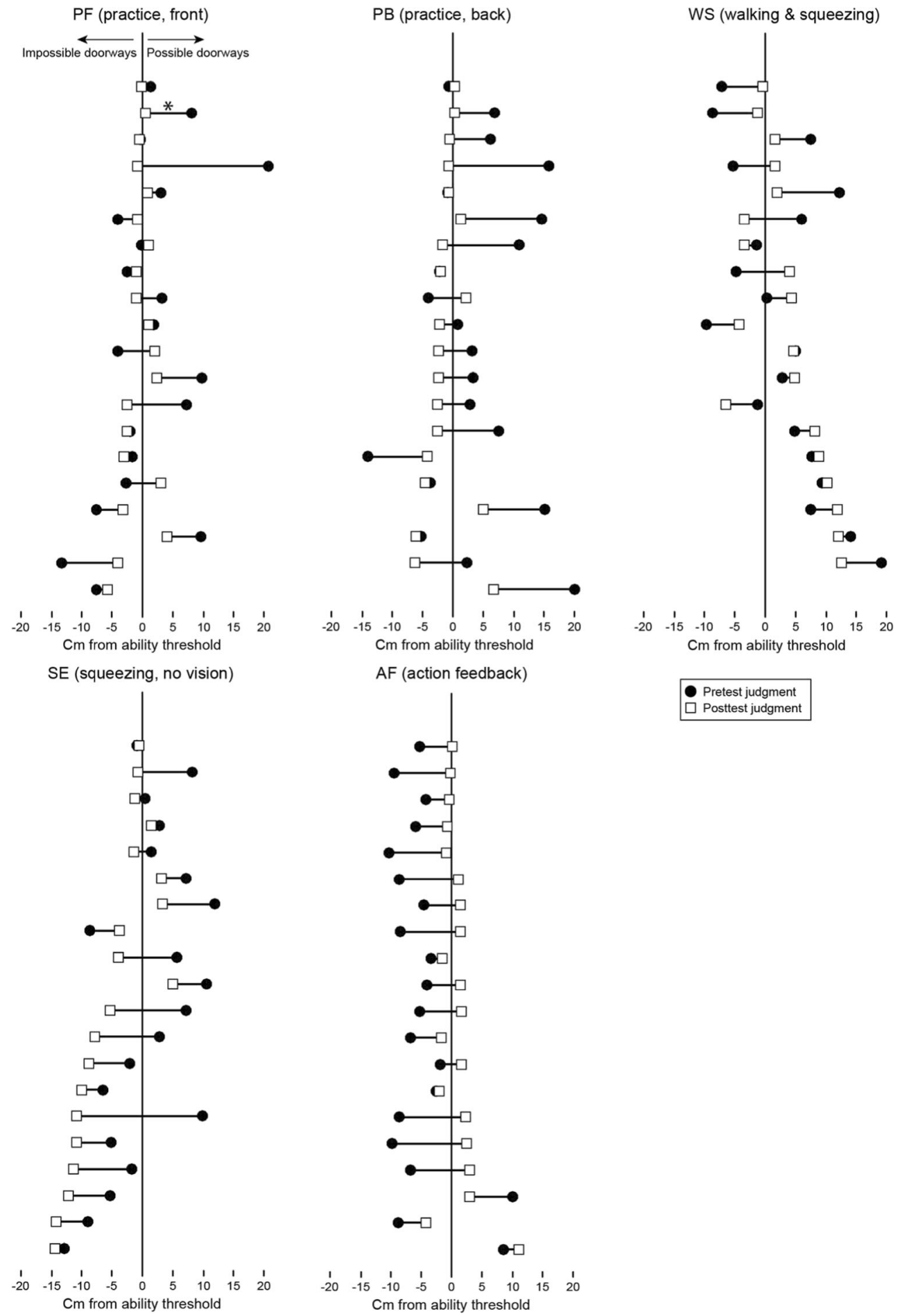

Fig. 3 Pretest judgment thresholds (circles) and posttest judgment thresholds (squares) for each participant in each condition. Judgments are plotted relative to each participant's backpack ability threshold ( $0 \mathrm{~cm}$ on the $x$-axis); values closer to $0 \mathrm{~cm}$ reflect judgments that are more accurate. Negative values indicate that participants erred by

judging that they could fit through impossibly small doorways, whereas positive values indicate that participants erred by judging that they could not fit through possible doorways. The asterisk (*) marks the participant whose data are shown in Fig. 2.

conditions $(p s=.99)$ or between the WS and SE conditions ( $p$ $=.99$ ).

Errors from one participant were outliers in the WS condition at both pretest $(26 \mathrm{~cm})$ and posttest $(31 \mathrm{~cm})$.

However, the significance of the main effects, interactions, and follow-up tests for the Condition $\times$ Phase ANOVA did not change when that participant's data were removed. 


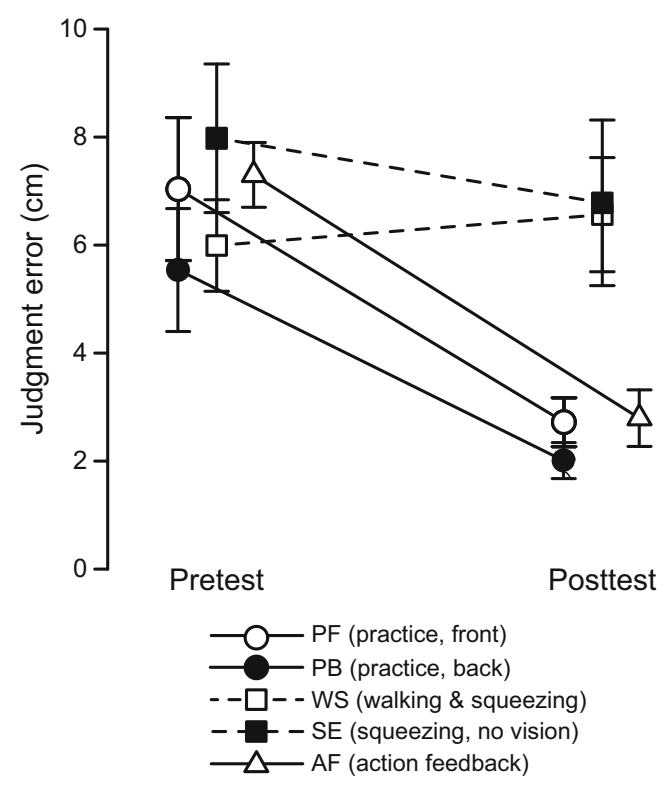

Fig. 4 Mean judgment errors before and after exploration in each condition. Error bars show one standard error.

\section{Vision of the backpack}

Direct comparison of errors between the practice (PF and $\mathrm{PB}$ ) conditions is misleading, because the manipulation sizes differed. Participants in the PB condition arguably had an easier task because their bodies were changed to a lesser degree while wearing the backpack on the back. To account for differences in manipulation size, proportional errors were calculated by dividing each participant's judgment errors by the manipulation size for that participant. Analyses of the proportional errors suggested a slight benefit of vision of the backpack: Participants in the PF condition recalibrated more fully than did participants in the PB condition. Whereas participants in the PB condition improved from $M=.96$ ( $S D$ $=.85)$ to $M=.35(S D=.28)$, participants in the PF condition improved from $M=.52(S D=.50)$ to $M=.19(S D=.16)$. A Condition $(\mathrm{PF}, \mathrm{PB}) \times$ Phase (pretest, posttest) ANOVA on proportional errors confirmed the main effects of phase, $F(1$, $38)=20.77, p<.001, \eta_{\mathrm{p}}{ }^{2}=.353$, and condition, $F(1,38)=$ $5.56, p=.024, \eta_{\mathrm{p}}{ }^{2}=.128$. The Condition $\times$ Phase interaction was nonsignificant $(p=.191)$. Sidak-corrected follow-ups indicated that participants in the PF condition were marginally more accurate at pretest $(p=.055)$ and significantly more accurate at posttest $(p=.027)$ than the participants in the PB condition.

\section{General discussion}

The present study tested whether exploratory behaviors that facilitate recalibration generalize among affordances for fitting through openings. Different types of exploratory behavior were assessed to determine whether each behavior allowed recalibration to changes in the affordances for squeezing through doorways. As in past work (Franchak \& Adolph, 2014b; Yasuda et al., 2014), practice walking through doorways (PF and $\mathrm{PB}$ conditions) improved affordance perception. Vision of the backpack alone was not a sufficient condition for recalibration but did lead to marginally better posttest accuracy following practice. Crucially, judgments did not improve after participants had walked around the room and pressed the backpack against the wall in the WS condition. Thus, the same exploratory behavior that recalibrates perception of fitting under barriers (locomotor experience that produces optic flow) does not necessarily generalize to squeezing through doorways. One interpretation of this result is that practice facilitated recalibration in the squeezing task because it provided action feedback. Consistent with this view, judgment feedback facilitated recalibration in the AF condition in the absence of walking and haptic experience.

\section{Evidence contradicts the function generalization hypothesis}

The function generalization hypothesis put forth in this article predicted that the same exploratory behaviors should support recalibration for functionally similar affordances. The present findings contradict this hypothesis. Locomotor experience in the WS condition, which recalibrated perception in the barrier task (Stoffregen et al., 2009), was insufficient for recalibration in the squeezing task (present study). Participants in the squeezing task recalibrated only after practicing squeezing through doorways or receiving judgment feedback. In those conditions, participants had visual information about doorway size coupled with feedback about action outcomes. It is important to note that the judgment feedback that participants received in the AF condition was not equivalent to the feedback generated during practice. Participants in the practice conditions generated feedback through their performance, and participants in the AF condition received feedback from the experimenter. Ideally, visual feedback would be isolated from the other types of information associated with practice (optic flow, haptic information, etc.). However, there is no way to separate visual feedback from the other types of visual information that participants experience during practice.

Whether feedback information was required for recalibration or was simply sufficient will be discussed in the next section. Regardless, locomotor experience was not found to be sufficient. This suggests that fitting affordances (walking through doorways, navigating under barriers, or squeezing through doorways) do not form a single category with respect to exploratory behaviors. More generally, the present findings cast doubt on the idea that functionally similar affordances always share the same means of exploration. This creates a 
challenging task for observers who are confronted with innumerable affordances in daily life: learning how to explore in order to recalibrate the perception of different types of affordances (including novel affordances). The present results suggest that observers lack a simple solution to this problem. Instead, they may need to learn many specific solutions to handle variations of similar affordances, and attempts to generalize exploratory processes to novel variants may fail. Indeed, past work showed that firefighters differed in their abilities to recalibrate two different fitting affordancessqueezing through doorways and walking under barrierswhile wearing bulky firefighting equipment (Petrucci et al., 2016). It is possible that this discrepancy stems from differences in how exploratory behaviors had recalibrated perception for each affordance.

Future research should study how observers spontaneously select exploratory behaviors in affordance tasks, to determine whether they choose behaviors appropriately. Whereas perceptual learning studies, such as those of dynamic touch (Riley, Wagman, Santana, Carello, \& Turvey, 2002; Turvey, 1996; Withagen \& Michaels, 2005), do not constrain exploration, studies of affordance perception in adults typically do. As in the present study, most affordance research assigns participants to explore or experience different types of information to measure effects on perception. Thus, it is unknown whether participants do generate the appropriate means of exploration. Ongoing work in my lab suggests that in the squeezing task, participants spontaneously choose to walk (thus producing optic flow) and attempt other haptic behaviors (e.g., touching the backpack or the doorway), even though those behaviors do not lead to improved perception in this task (Labinger, Monson, \& Franchak, 2017).

\section{The role of practice and feedback in affordance perception}

The most parsimonious explanation of the present results is that practice facilitates recalibration because action feedback is required. Participants only recalibrated in conditions with feedback about the action outcomes. This is consistent with the work of Yasuda and colleagues (2014), who showed that participants learned following practice only when they experienced both successful and failed outcomes. Exposure only to successful outcomes did not permit learning, perhaps because participants could not determine the point at which they would fail. In other words, success-only feedback failed to calibrate perception of doorway width relative to body size.

However, the present study and past work (Franchak \& Adolph, 2014b; Yasuda et al., 2014) were unable to eliminate two alternatives that preclude making such a strong claim about what information is required for recalibration. One possibility is that exploratory behaviors other than the ones tested could also have led to recalibration (thus still contradicting the function generalization hypothesis). The present findings provide an important first step by ruling out some possibilities. Neither optic flow from postural sway while standing nor optic flow from self-produced locomotion, despite leading to accurate perception for other affordances (Fath \& Fajen, 2011; Mark, 1987; Mark et al., 1990; Stoffregen et al., 2005; Stoffregen et al., 2009; Yu et al., 2011; Yu \& Stoffregen, 2012), was sufficient. Neither was haptic information from squeezing in the WS and SE conditions, despite the facilitative role played by haptic exploration in other affordance tasks (Joh et al., 2007; Wagman \& Hajnal, 2014, 2016). Although visual and haptic information combined from walking across the room and compressing the backpack against the wall was not sufficient, some other form of exploration that was not tested in the present studies might yet be sufficient, alone or in combination with the exploratory behaviors that were tested. Many variations might need to be tested before another sufficient condition is observed. For example, despite decades of research on the perception of length through dynamic touch, only recently have researchers discovered means of improving the perception of length solely through haptic exploration, as opposed to visual and haptic information or haptic information augmented by judgment feedback (Abney, Wagman, \& Schneider, 2014; Stephen \& Arzamarski, 2009).

A second possibility is that the walking and squeezing experience that was tested could have led to recalibration if participants had received more of the same experience. For another affordance, brief practice was insufficient for novice wheelchair users to accurately judge their abilities to roll through doorways that varied in width (Higuchi, Takada, Matsuura, \& Imanaka, 2004; Yasuda et al., 2014). Practicing over eight days reduced errors, but not completely (Higuchi et al., 2004). However, the numbers of walking and squeezing trials were chosen to match the amount of practice participants had received in prior investigations (Franchak \& Adolph, 2014b; Franchak et al., 2010; Yasuda et al., 2014). Furthermore, the amount of walking experience was $75 \%$ greater than the amount that had been sufficient for calibrating perception of fitting under barriers (Stoffregen et al., 2009).

Thus, more work will be needed to distinguish whether action feedback is a necessary condition for recalibration for squeezing through doorways, or whether it is only a sufficient condition. Testing other exploratory behaviors and varying the amount of experience will help discern between these possibilities.

If it is the case that participants are only able to learn from practice, why might some affordances depend on action feedback and others do not? Most likely it depends on the information for perceiving affordances and how that information is altered by changes to the body and abilities. Practicing the action is not necessary to perceive whether it is possible to step on risers (Mark, 1987; Warren, 1984) or sit on seats (Mark et al., 1990) and to navigate under barriers (Franchak et al., 2012; Wagman \& Malek, 2009). Practice is sufficient for recalibration to rolling under barriers in a wheelchair, but is 
no better than visual information from self-produced locomotion (Stoffregen et al., 2009). A key similarity in the studies above is that perception depends on eye-height-scaled information in optic flow patterns. Furthermore, changes to the body that alter affordances - for instance, wearing platform shoes or sitting in a wheelchair - change eye height at the same time.

In contrast, wearing a backpack or pregnancy pack (Franchak \& Adolph, 2014b) alters affordances without changing visual information. Similarly, firefighters inaccurately judged affordances for crouching under a barrier when wearing a large breathing apparatus on their backs (Petrucci et al., 2016). For such changes to the body that do not alter visual information, practice might be an important form of exploration if observers need action feedback to determine how their affordances have changed. That wearing the backpack on the front of the body rather than the back altered affordances by a different degree (discussed below) highlights the complexities of the body-object-environment relations that determine affordances. Accordingly, the information required to perceive complex affordances (involving both body size and the dynamics of body compression) may be equally complex. There is precedent for affordance calibration depending on feedback: Visual feedback from throwing actions was needed to calibrate the perception of throwability (Zhu \& Bingham, 2010). Practicing throwing is the only way to generate feedback about throwing distance. A better understanding of which types of information are sufficient for perceiving a wider range of affordances should provide insights into the processes by which exploration supports affordance perception and recalibration. More research will be needed to understand the role of practice in other tasks, like leaping (Cole et al., 2013; Day et al., 2015) and brachiating (Cole et al., 2013). Practice might be important for providing feedback or might generate other useful types of information.

Another unanswered question is what it means to perceive an affordance accurately. How much error is acceptable? In the present study, I called perception inaccurate if there was room for improvement. Because participants in the practice and feedback conditions managed to reduce errors to only 2 $3 \mathrm{~cm}$, I concluded that their pretest errors of 5-8 $\mathrm{cm}$ reflected inaccurate perception. But there is no consensus in the literature as to what it means for affordance perception to be accurate. This is not merely a methodological issue. More research will be needed to understand whether such different levels of accuracy produce meaningful effects on functional behavior: How accurate must affordance perception be to support the activities of daily life?

\section{Vision of the backpack}

Vision of the backpack improved accuracy but was neither necessary nor sufficient for recalibration. Participants in both practice conditions successfully recalibrated, but posttest judgments with vision of the backpack were slightly more accurate than those without (when considering proportional accuracy). Seeing the backpack may have also helped participants in the PF condition make slightly more accurate judgments during pretest trials. Finding a benefit of vision of the backpack was unexpected given that past work demonstrated perceptual independence between affordance judgments and metric judgments of objects that manipulated affordances (Mark, 1987; Thomas \& Riley, 2014; Thomas et al., 2017). However, these benefits of visual information about the backpack should be interpreted cautiously because differences between vision conditions were only observed when measuring accuracy as a proportion of manipulation size. Future work that equates manipulation size across vision conditions can better address the role of visual information about a bodymodifying object.

It was surprising to find that wearing the backpack on the front of the body led to a greater change in ability thresholds (manipulation size) than did wearing the backpack on the back. Possibly, the larger manipulation size in the PF condition resulted from differences in body topography. On the front of the body, the abdomen is the farthest protruding part of many participants' torsos. Placing the backpack over the abdomen maximizes its effect. For the back of the body, the buttocks protrude the farthest. Thus, when the backpack is worn on the middle of the back, the backpack's effect of increasing ability thresholds relative to baseline might be diminished. Were the backpack to be placed, instead of the middle of the back, on the buttocks (in what would be a particularly awkward manipulation), the manipulation sizes might be equated between the conditions. However, this explanation is muddied by unknown variability in body topography. These speculations could not be verified because detailed anthropometric measurements were not collected.

\section{Conclusion}

The ecological psychology literature points to multiple sources of information that support perception for a wide range of affordances. What works for perceiving one affordance may not necessarily work for another: Information that supports perception varies according to the nature of the actor-environment fit of a particular action. Thus, how actors need to explore to generate that information may also be specific to different affordances. The present findings reveal that this specificity may arise even within categories of functionally similar affordances, in which slight task variations create large shifts in exploratory processes. Future work will further clarify the difficulty of the problem faced by observers in learning to perceive complex, real-world affordances and assess the effectiveness of observers' solutions. 
Author note I thank Eli Labinger, Ria Madon, Corey Mize, and the other members of the UCR Perception, Action, and Development lab for their assistance on this project. I am grateful to Iman Feghhi, Kari Kretch, and David Rosenbaum for providing helpful comments on earlier versions of the manuscript.

\section{References}

Abney, D. H., Wagman, J. B., \& Schneider, W. J. (2014). Changing grasp position on a wielded object provides self-training for the perception of length. Attention, Perception, \& Psychophysics, 76, 247-254.

Adolph, K. E. (1995). Psychophysical assessment of toddlers' ability to cope with slopes. Journal of Experimental Psychology: Human Perception and Performance, 21, 734-750.

Adolph, K. E. (1997). Learning in the development of infant locomotion. Monographs of the Society for Research in Child Development, 62(Serial No. 251), 1-158.

Adolph, K. E. (2008). Learning to move. Current Directions in Psychological Science, 17, 213-218.

Adolph, K. E., Joh, A. S., Franchak, J. M., Ishak, S., \& Gill-Alvarez, S. V. (2008). Flexibility in the development of action. In J. Bargh, P. Gollwitzer, \& E. Morsella (Eds.), The psychology of action (Vol. 2, pp. 399-426). New York, NY: Oxford University Press.

Bingham, G. P., \& Pagano, C. C. (1998). The necessity of a perceptionaction approach to definite distance perception: Monocular distance perception to guide reaching. Journal of Experimental Psychology: Human Perception and Performance, 24, 145-168.

Bingham, G. P., Schmidt, R. C., \& Rosenblum, L. D. (1989). Hefting for a maximum distance throw: A smart perceptual mechanism. Journal of Experimental Psychology: Human Perception and Performance, 15, 507-528. doi:10.1037/0096-1523.15.3.507

Bruggeman, H., \& Warren, W. H. (2010). The direction of walking-but not throwing or kicking - is adapted by optic flow. Psychological Science, 21, 1006-1013.

Cole, W. G., Chan, G. L. Y., \& Adolph, K. E. (2013). Perceiving affordances for different motor skills. Experimental Brain Research, 225, 309-319.

Comalli, D. M., Franchak, J. M., Char, A., \& Adolph, K. E. (2013). Ledge and wedge: Younger and older adults' perception of action possibilities. Experimental Brain Research, 228, 183-192. doi:10.1007/ s00221-013-3550-0

Cornsweet, T. N. (1962). The staircase-method in psychophysics. American Journal of Psychology, 75, 485-491.

Day, B. M., Wagman, J. B., \& Smith, P. J. K. (2015). Perception of maximum stepping and leaping distance: Stepping affordances as a special case of leaping affordances. Acta Psychologica, 158, 2635.

Fath, A. J., \& Fajen, B. R. (2011). Static and dynamic visual information about the size and passability of an aperture. Perception, 40, 887904.

Franchak, J. M., \& Adolph, K. E. (2014a). Affordances as probabilistic functions: Implications for development, perception, and decisions for action. Ecological Psychology, 26, 109-124.

Franchak, J. M., \& Adolph, K. E. (2014b). Gut estimates: Pregnant women adapt to changing possibilities for squeezing through doorways. Attention, Perception, \& Psychophysics, 76, 460-472. doi:10.3758/ s13414-013-0578-y

Franchak, J. M., Celano, E. C., \& Adolph, K. E. (2012). Perception of passage through openings cannot be explained geometric body dimensions alone. Experimental Brain Research, 223, 301-310.

Franchak, J. M., van der Zalm, D., \& Adolph, K. E. (2010). Learning by doing: Action performance facilitates affordance perception. Vision Research, 50, 2758-2765.
Franchak, J. M., van der Zalm, D. J., Hartzler, B. M., \& Adolph, K. E. (2009, July). Perceiving affordances for navigating through openings. Paper presented at the International Conference on Perception and Action, Minneapolis, MN.

Gibson, J. J. (1979). The ecological approach to visual perception. Boston, MA: Houghton Mifflin.

Higuchi, T., Murai, G., Kijima, A., Seya, Y., Wagman, J. B., \& Imanaka, K. (2011). Athletic experience influences shoulder rotations when running through apertures. Human Movement Science, 30, 534-549. doi:10.1016/j.humov.2010.08.003

Higuchi, T., Takada, H., Matsuura, Y., \& Imanaka, K. (2004). Visual estimation of spatial requirements for locomotion in novice wheelchair users. Journal of Experimental Psychology: Applied, 10, 5566. doi:10.1037/1076-898X.10.1.55

Joh, A. S., Adolph, K. E., Campbell, M. R., \& Eppler, M. A. (2006). Why walkers slip: Shine is not a reliable cue for slippery ground. Perception \& Psychophysics, 68, 339-352.

Joh, A. S., Adolph, K. E., Narayanan, P. J., \& Dietz, V. A. (2007). Gauging possibilities for action based on friction underfoot. Journal of Experimental Psychology: Human Perception and Performance, 33, 1145-1157.

Kingdom, F. A. A., \& Prins, N. (2010). Psychophysics: A practical introduction. New York, NY: Academic Press.

Kretch, K. S., \& Adolph, K. E. (2013). Cliff or step? Posture-specific learning at the edge of a drop-off. Child Development, 84, 226-240. doi:10.1111/j.1467-8624.2012.01842.x

Labinger, E., Monson, J., \& Franchak, J. M. (2017). Spontaneous selection of exploratory behaviors while recalibrating to altered affordances for squeezing through doorways. Manuscript in preparation.

Mark, L. S. (1987). Eyeheight-scaled information about affordances: A study of sitting and stair climbing. Journal of Experimental Psychology: Human Perception and Performance, 13, 361-370.

Mark, L. S., Baillet, J. A., Craver, K. D., Douglas, S. D., \& Fox, T. (1990). What an actor must do in order to perceive the affordance for sitting. Ecological Psychology, 2, 325-366.

Michaels, C. F., \& Oudejans, R. R. (1992). The optics and actions of catching fly balls: Zeroing out optical acceleration. Ecological Psychology, 4, 199-222.

Oudejans, R. R., Michaels, C. F., Bakker, F. C., \& Dolne, M. A. (1996). The relevance of action in perceiving affordances: Perception of catchableness of fly balls. Journal of Experimental Psychology: Human Perception and Performance, 22, 879-891.

Petrucci, M. N., Horn, G. P., Rosengren, K. S., \& Hsiao-Wecksler, E. (2016). Inaccuracy of affordance judgments for firefighters wearing personal protective equipment. Ecological Psychology, 28, 108126.

Rieser, J. J., Pick, H. L., Ashmead, D. H., \& Garing, A. E. (1995). Calibration of human locomotion and models of perceptual-motor organization. Journal of Experimental Psychology: Human Perception and Performance, 21, 480-497.

Riley, M. A., Wagman, J. B., Santana, M. V., Carello, C., \& Turvey, M. T. (2002). Perceptual behavior: Recurrence analysis of a haptic exploratory procedure. Perception, 31, 481-510.

Stephen, D. G., \& Arzamarski, R. (2009). Self-training of dynamic touch: Striking improves judgment by wielding. Attention, Perception, \& Psychophysics, 71, 1717-1723.

Stoffregen, T. A. (2003). Affordances as properties of the animalenvironment system. Ecological Psychology, 15, 115-134.

Stoffregen, T. A., Yang, C., \& Bardy, B. G. (2005). Affordance judgments and nonlocomotor body movement. Ecological Psychology, 17, 75104.

Stoffregen, T. A., Yang, C., Giveans, M. R., Flanagan, M., \& Bardy, B. G. (2009). Movement in the perception of an affordance for wheelchair locomotion. Ecological Psychology, 21, 1-36. 
Thomas, B. J., \& Riley, M. A. (2014). Remembered affordances reflect the fundamentally action-relevant, context-specific nature of visual perception. Journal of Experimental Psychology: Human Perception and Performance, 40, 2361-2371.

Thomas, B. J., Wagman, J. B., Hawkins, M., Havens, M., \& Riley, M. A. (2017). The independent perceptual calibration of action-neutral and -referential environmental properties. Perception, 46, 586-604. doi: 10.1177/0301006616679172

Turvey, M. T. (1996). Dynamic touch. American Psychologist, 51, 1134 1152.

Wagman, J. B., \& Hajnal, A. (2014). Task specificity and anatomical independence in perception of properties by means of a wielded object. Journal of Experimental Psychology: Human Perception and Performance, 40, 2372-2391.

Wagman, J. B., \& Hajnal, A. (2016). Use your head! Perception of action possibilities by means of an object attached to the head. Experimental Brain Research, 234, 829-836.

Wagman, J. B., \& Malek, E. (2009). Geometric, kinetic-kinematic, and intentional constraints influence willingness to pass under a barrier. Experimental Psychology, 56, 409-417.

Wagman, J. B., \& Taylor, K. R. (2005). Perceiving affordances for aperture crossing for the person-plus-object system. Ecological Psychology, 17, 105-130.

Warren, W. H. (1984). Perceiving affordances: Visual guidance of stair climbing. Journal of Experimental Psychology: Human Perception and Performance, 10, 683-703.

Warren, W. H., \& Whang, S. (1987). Visual guidance of walking through apertures: Body-scaled information for affordances. Journal of
Experimental Psychology: Human Perception and Performance, 13, 371-383.

Wichmann, F. A., \& Hill, N. J. (2001a). The psychometric function: I. Fitting, sampling, and goodness of fit. Perception \& Psychophysics, 63, 1293-1313. doi:10.3758/BF03194544

Wichmann, F. A., \& Hill, N. J. (2001b). The psychometric function: II. Bootstrap-based confidence intervals and sampling. Perception \& Psychophysics, 63, 1314-1329. doi:10.3758/BF03194545

Withagen, R., \& Michaels, C. F. (2005). The role of feedback information for calibration and attunement in perceiving length by dynamic touch. Journal of Experimental Psychology: Human Perception and Performance, 31, 1379-1390. doi:10.1037/0096-1523.31.6. 1379

Yasuda, M., Wagman, J. B., \& Higuchi, T. (2014). Can perception of aperture passability be improved immediately after practice in actual passage? Dissociation between walking and wheelchair use. Experimental Brain Research, 232, 753-764.

Yu, Y., Bardy, B. G., \& Stoffregen, T. A. (2011). Influences of head and torso movement before and during affordance perception. Journal of Motor Behavior, 43, 45-53.

Yu, Y., \& Stoffregen, T. A. (2012). Postural and locomotor contributions to affordance perception. Journal of Motor Behavior, 44, 305-311.

Zhu, Q., \& Bingham, G. P. (2010). Learning to perceive the affordance for long-distance throwing: Smart mechanism or function learning? Journal of Experimental Psychology: Human Perception and Performance, 36, 862-875. 